\title{
Telematics for the Analysis of Vehicle Fleet Fuel Consumption
}

\author{
Wolfgang Niebel ${ }^{1}$, Rüdiger Ebendt ${ }^{1}$, and \\ Günter Kuhns ${ }^{1}$ \\ ${ }^{1}$ German Aerospace Center (DLR), Institute of Transportation Systems, Department of \\ Traffic Management, Rutherfordstr. 2, 12489 Berlin, Germany \\ \{Wolfgang.Niebel; Ruediger.Ebendt; Guenter.Kuhns\}@dlr.de
}

\begin{abstract}
Within the European research project SimpleFleet, a telematics system for fleet management business intelligence was developed, delivering live or near-realtime statistics about individual vehicles or the entire fleet. This supports the fleet manager in ad-hoc decisions and in medium and long term improvements. The fleet vehicles (e.g. taxis, delivery vans) are equipped with an On-Board-Unit (OBU) for GPS tracking and data collection from other sensors, e.g., an engine on/off detector. These raw data are sent to a host system and database, being enhanced to Floating Car Data (FCD) using map-matching and routing. Beside other route-related information, consulted fleet operators expressed their interest especially in fuel consumption calculation. For this purpose the German EWS procedure was chosen, providing 2nd degree polynomial functions of the driven speed. Final results for the test in the Athens region are presented, including the comparison of two calculation approaches with different aggregation levels of driven speeds.
\end{abstract}

Keywords: Logistic services, Fleet Management, Floating Car Data.

\section{Introduction}

Project SimpleFleet makes it easy for small and medium enterprises (SMEs), both, from a technological and business perspective, to create web-based fleet management applications. For this purpose, a large data pool ("TrafficStore") comprising base data such as maps and traffic data from dedicated providers has been built. A simple interface provides a means to connect user-contributed data streams to this pool. An algorithmic framework dubbed "TrafficIntelligence" which includes map-matching algorithms, vehicle routing services and a statistics package utilizes the collected data and provides value-added services. SMEs are able to access the data and services by means of a web-based API, a Software Development Kit (SDK) wrapping API access for specific languages and environments and Application Frameworks for rapid application development for target platforms such as Web (JavaScript), and iPhone and Android mobile platforms.

To ensure a service development which addresses the real needs of the SMEs a Customer Advisory Board was set up. From their feedback it became clear that adding a sensor to measure the fuel consumption is possible, but too expensive, so most fleet managers would probably not pay for it. Instead they calculate the fuel consumption, based only on the recorded speeds. To overcome this vague approach a 
more sophisticated method was developed as described in section 2. Its implementation (section 3) was tested with field data from a member of the advisory board, B-K-Telematics. This telematics and fleet management service provider is settled in the urban area of Athens/Greece. Section 4 gives some results from that test. Detailed descriptions of the work are contained in [1].

\section{Fleet Analytics Methodology}

\subsection{Input Data}

Raw data is delivered by the original fleet management systems into the TrafficStore via the TrafficAPI. It contains series of time stamped geo-coordinates (traces) derived from the GPS functionality of the On-Board-Units. B-K Telematics provided the following additional information per GPS trace:

- $\quad$ vehicle type,

- $\quad$ engine on/off to identify stops, and

- $\quad$ engine on but stopped (which fleet managers will consider as a rather undesired state).

\subsection{Basic Performance Indicator Computation}

Basic performance indicators desired by the Customer Advisory Board comprise the kilometrage and number of stops.

Kilometrage calculation requires some pre-processing steps which are wellestablished within the field of Floating Car Data (FCD). These algorithms enhance the received geo-traces by map-matching and routing based on road network maps. Summing up the lengths of these trajectories yields the kilometrage.

A vehicle is considered as stopped when its engine is off. The most important attributes defining a stop include the identity of the vehicle, the time the vehicle stopped and the time the vehicle started moving again. Additionally, the position of the vehicle at the start time point and the position at the end time point of a stop are also considered as attributes of a stop. The data model used for storing vehicle stops for FleetAnalytics is simple and is realized using one relational table in a PostgreSQL database (DB). The population of the stops $\mathrm{DB}$ table is triggered from the FCD collection process. A database trigger defined on the INPUT_FCD_FLEET DB populates the stops table based on fact that there are two possible conditions for one vehicle:

- $\quad$ Condition A: If one vehicle is currently stopped, then its most recent entry in the stops table contains the start time point of the stop and a null value for the end time point.

- $\quad$ Condition B: If a vehicle is currently moving, then its most recent entry in the stops table contains the start and end time points of the last stop realized by the particular vehicle. There is also the special case that no record is contained in the stops table for the particular vehicle. This case appears only 
once for one vehicle, at the first time it is encountered by the stops population process.

For a new FCD sample that is considered by the database trigger, the following actions may be realized:

- If Condition A is true and the FCD sample's engine status value is ON, then the end time point of the most recent record in the stops table, for the particular vehicle producing this FCD sample, is updated using the timestamp value of the FCD sample.

- If Condition B is true and the FCD sample's engine status value is OFF, then a new entry is stored in the stops table for this particular vehicle. The start time point of the record gets the value of the FCD sample's timestamp and the end time point is set to NULL.

\subsection{Fuel Consumption Model}

An appropriate fuel consumption model had to be chosen, for which the decision criteria were, i.a., suitability for the existing data range and format like vehicle type and speed, as well as its applicability in Europe. Within the EU FP7 project iTetris ("An Integrated Wireless and Traffic Platform for Real-Time Road Traffic Management Solutions”), literature regarding the models for pollutant emission and fuel consumption has been reviewed [2]. A lot of the therein found models turned out to be not suitable for SimpleFleet, as it became clear that a large amount of static parameters such as the engine displacement, and parameters modelling the processes within the vehicle's engine should not be expected to be available. At the end the German EWS sub-model for fuel consumption [3] was chosen.

It is particularly the ability of EWS to deliver continuous functions which makes an advantage over COPERT III [4] or HBEFA [5]. Nonetheless, it is possible to use both the (online) FLEET data of, e.g., EMISIA S.A. [6], and the most recent HBEFA publications to adapt the factors of EWS for a particular forecast period and for most European countries.

The dependency of fuel consumption $k$ on

- (mean) velocity V $(\mathrm{km} / \mathrm{h}]$

- longitudinal slope s [\%] (positive: ascending, negative: descending)

- considered year Y

is modeled by a multiplicative conjunction as

$$
k=\mathrm{F}(\mathrm{V}) \cdot \mathrm{G}(\mathrm{s}, \mathrm{V}) \cdot \mathrm{H}(\mathrm{Y})\left[\mathrm{g} / \mathrm{veh}^{*} \mathrm{~km}\right] .
$$

The functions $G$ and $H$ are normed such that $G(s=0, V)=1.0$ for slope $s=0 \%$, and such that $\mathrm{H}(\mathrm{Y}=1990)=1.0$ for the base year . The respective empiric values represent mean values of the measurements for classes of engine displacement, gross vehicle weight, etc.

Note that the approach taken here is more general than necessary for some customers of SimpleFleet's FleetAnalytics suite of software modules, since information about the longitudinal slope might not be at hand in the road network map. Due to $G(s=0, V)=1.0$, this is not a problem for the model. 
Regarding function $\mathrm{F}$, it is of note that the mean velocity $\mathrm{V}$ is used to distinguish between two patterns of driver behavior. One is the pattern of "normal range of travel speeds", the other is the "stop-and-go pattern". The first is always detected when $\mathrm{V}>20 \mathrm{~km} / \mathrm{h}$, and the latter is detected when $\mathrm{V} \leq 20 \mathrm{~km} / \mathrm{h}$. Fuel consumption in this case is the minimum of either a constant "stop-and-go factor" $\mathrm{c}_{\mathrm{s}}$ or the calculated value for the "normal range of travel speeds". Written as a formula, we have that

$$
F(V)=\left\{\begin{array}{c}
c_{0}+c_{1} \cdot V^{2}+\frac{c_{2}}{V} \text { for } V>20 \mathrm{~km} / \mathrm{h} \\
\min \left\{c_{s},\left(c_{0}+c_{1} \cdot V^{2}+\frac{c_{2}}{V}\right)\right\} \text { for } V \leq 20 \mathrm{~km} / \mathrm{h}
\end{array}\right.
$$

The values $c_{s}, c_{0}, c_{1}, c_{2}$ can be found in look-up tables [3] for each of the eight EWS vehicle classes, e.g., $\mathrm{PO}=$ Passenger car with Otto engine (petrol), $\mathrm{LN}=$ light duty etc., differentiated between urban roads and freeways. A final conversion from grams into liters can be applied if needed.

It could be proved by fleet mileage data for the year 2005 [6] that the Greek fleet's specific consumption (per vehicle) is a few percent less than the German fleet's (max. 10\%). Therefore the EWS parameters were found to be eligible for use for Greece. Since no data for future fuel consumption in Greece was available, the respective trends in Germany were assumed to be valid again. HBEFA [5] data for fuel consumption is available from 1995 on until the year 2035. For each vehicle the relative change $\mathrm{H}(\mathrm{Y})$ was derived for the years 2014 to 2025 as stated in Table 1. It is noteworthy that for duty vehicles no decreases or even slight increase are expected between 2014 and 2025 according to these data.

Table 1: Correction factors $\mathrm{H}(\mathrm{Y})$ for the EWS vehicle types, exemplary years

\begin{tabular}{lllllll}
\hline \multirow{2}{*}{ EWS vehicle type ID (according B-K fleet vehicle type) } & \multicolumn{5}{c}{ Year Y } \\
\cline { 3 - 7 } & & 1990 & 2014 & $2015 \ldots$ & $2020 \ldots$ & 2025 \\
\hline PO (passenger car) & 1.00 & 0.77 & 0.76 & 0.70 & 0.64 \\
LN (van 4X4 Pickup) & 1.00 & 0.75 & 0.73 & 0.62 & 0.52 \\
\multirow{2}{*}{ ZS (refrigerator truck) } & urban road & 1.00 & 0.89 & 0.89 & 0.91 & 0.92 \\
& freeway & 1.00 & 0.96 & 0.96 & 0.97 & 0.97 \\
\hline
\end{tabular}

\section{Implementation}

A prototype of the FleetAnalytics suite of software modules has been implemented in Java by DLR. It operates on a test fleet of $\sim 400$ vehicles in Athens as provided by B$\mathrm{K}$ Telematics. The implemented approach to calculate the fuel consumption is based on map-matched trajectories of the FCD data. The speed of an individual vehicle is derived from the time-stamped position reports of its trajectories. The trajectories are stored in a database where each trajectory consists of a chain of trajectory entries containing a timestamp, an edge identifier, the edge length, the edge speed and a flag that identifies motorways. 
Because of the map-matched FCD data we now have information about the type of the road and can apply special formula constants for motorways to achieve better results.

In a nutshell the implemented algorithm should calculate:

- The sum of the fuel consumption of the whole day over all vehicles in liters.

- The travelled distance of all vehicles for the whole day as sum of kilometers. Next, a description of the steps of the algorithm based on trajectories is given:

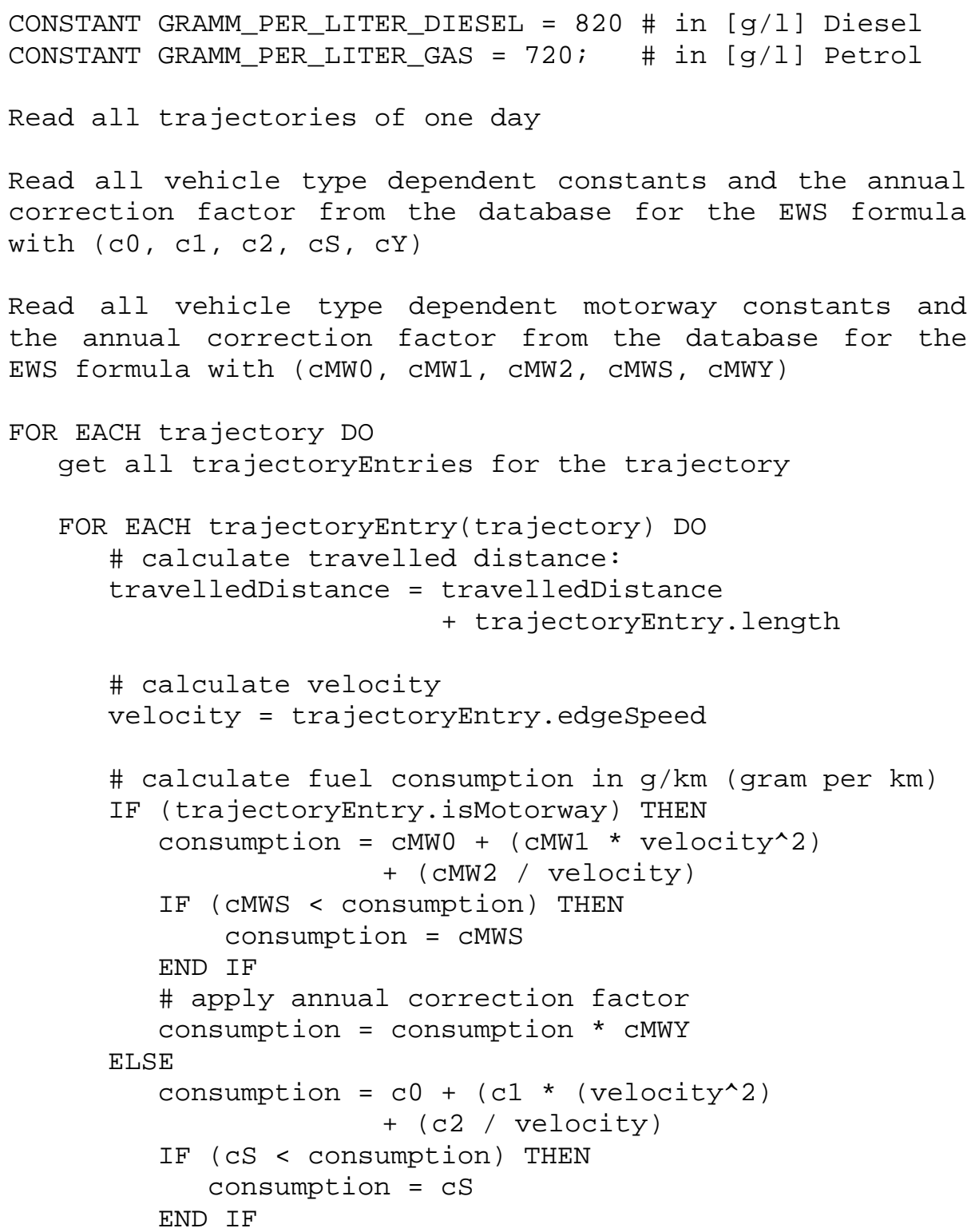

J. Mikulski (Ed.): TST 2014, CCIS 471, pp. 461-468, 2014 @ Springer-Verlag Berlin Heidelberg 2014 


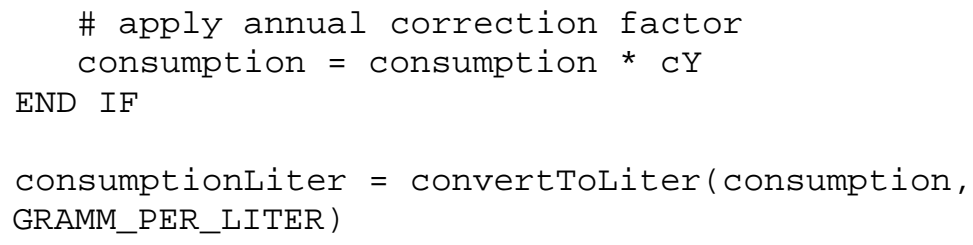

\section{Experimental Results}

To demonstrate the operation of the FleetAnalytics prototype, several statistics have been calculated, based on an exemplary week of fleet operation. The week chosen was from Monday the 3rd June until Sunday the 9th June 2013. The herein given statistics are for the whole fleet, rather than for particular individual vehicles, which is also enabled by the FleetAnalytics suite of software modules. The aggregated view should give a more interesting and more instructive experimental result. Fig. 1 gives the average fuel consumption per day and vehicle type, clearly revealing that the refrigerator trucks consume the most fuel on average. The two weekend days can also be identified by the absence of passenger cars (SUV). There seems to be no day-type dependencies of the specific fuel consumption.

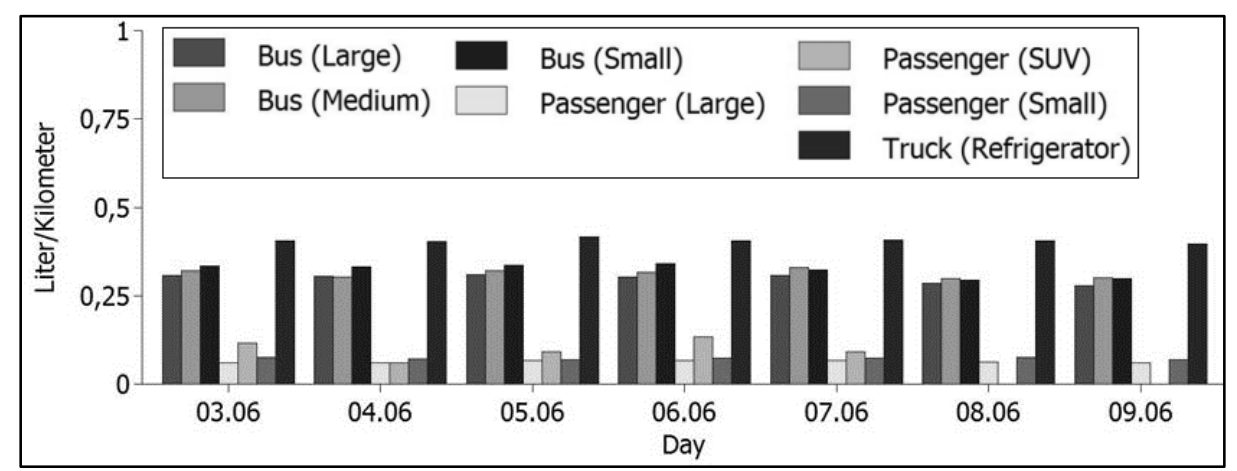

Fig. 1: Average fuel consumption per day and vehicle type in June 2013

In Fig. 2, the distribution of the overall fuel consumption per vehicle type of the test fleet over daytime is depicted (limited to the period of interest; after 2:30 pm the overall level remains for another 3 hours and further decreases with time). We can 
identify an early consumption peak between 3 am and 5:30 am, i.e., before the morning rush hour. The other consumption peak between noon and 2:30 pm is also before the afternoon rush hour. Refrigerator trucks are very active between midnight and the first peak as well as after the first peak but cease operation even before noon. Passenger cars do not have a significant share in the overall consumption.

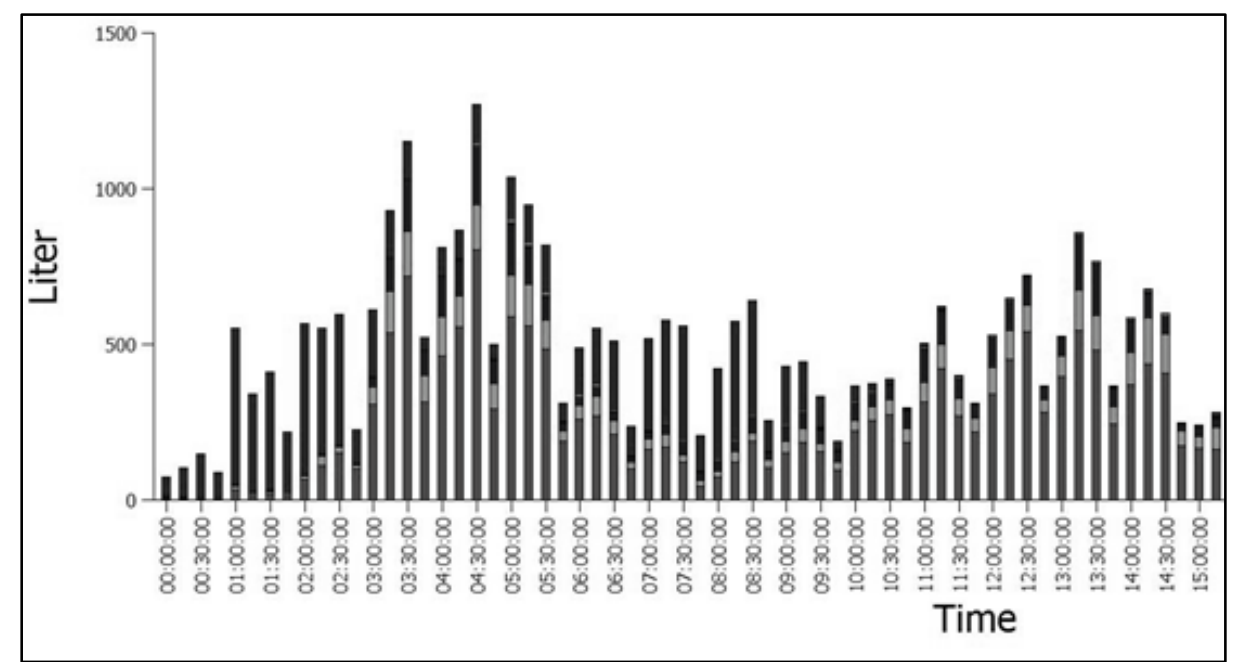

Fig. 2: Absolute fuel consumption per 15 minutes

The velocity distribution in Fig. 3 shows a peak at 20 to $25 \mathrm{~km} / \mathrm{h}$, and a second one at $80 \mathrm{~km} / \mathrm{h}$, mainly for refrigerator trucks. This might be due to their speed limit which is lower than the limit for buses at $100 \mathrm{~km} / \mathrm{h}$. Another reason could be the more frequent usage of freeways.

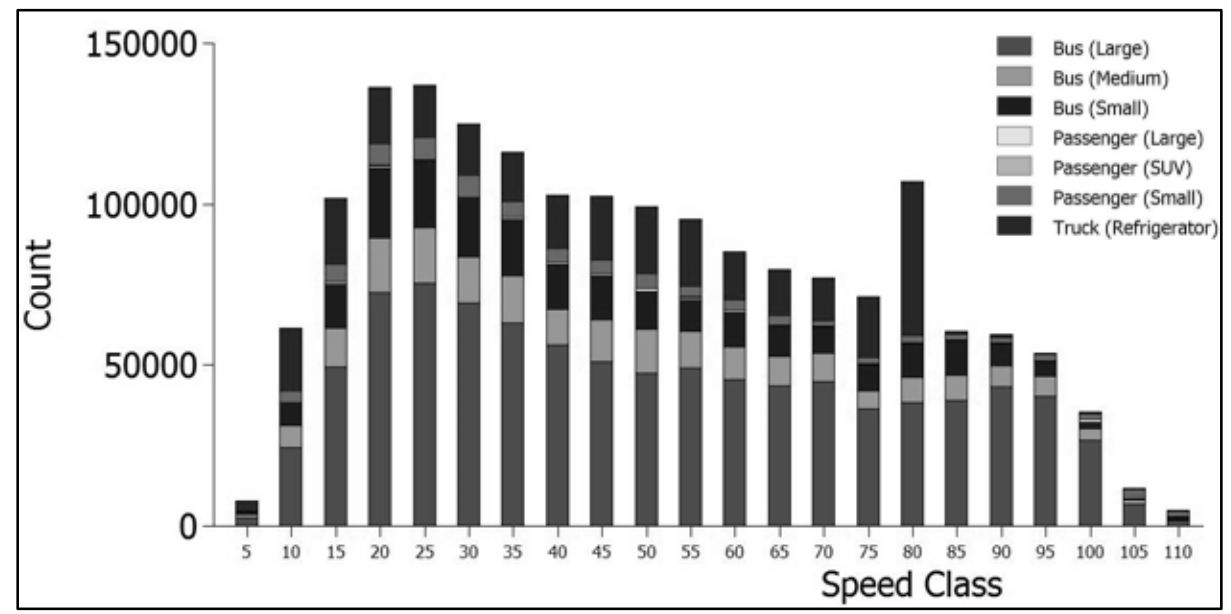

Fig. 3: Distribution of velocities by vehicle types, week from 03 June to 09 June 2013 


\section{Conclusion and Outlook}

The software suite for the business intelligence of the project SimpleFleet has been described. The FleetAnalytics suite is capable of calculating important daily statistics for the tracked assets of connected fleet managers, such as the fuel consumption. It is now up to the companies to use this new tool and gain experience how to utilize it for improvements. Such an improvement could be Eco-Routing by including not only travel times but also fuel consumption (and therefore emissions) into their routing algorithms.

Acknowledgments. The research leading to these results has received funding from the European Union Seventh Framework Programme "SimpleFleet" (http://www.simplefleet.eu, grant agreement No. FP7-ICT-2011-SME-DCL-296423). The authors like to thank RC Athena in Athens for the collaboration in providing fleet data.

\section{References}

1. Ebendt, R., Sauerländer-Biebl, A., Kuhns, G., Niebel, W., Efentakis, A., Magenschab, G.: SimpleFleet - Democratizing Fleet Management, Deliverable 3.2: Business Intelligence (Confidential). Berlin (2013)

2. iTETRIS consortium: Deliverable D3.1 - Traffic Modelling: Environmental Factors, (2009), http://ict-itetris.eu

3. P. Cerwenka, N. Dischinger, M. Klamer: Anwendungsorientierte Ermittlung von Kraftstoffverbrauch und Schadstoffemissionen des Kraftfahrzeugverkehrs in Deutschland für die Neufassung der RAS-W (EWS). In: Wirtschaftlichkeitsuntersuchungen an Straßen - Stand und Entwicklung der EWS, p. 15-23, Forschungsgesellschaft für Straßen- und Verkehrswesen (FGSV), Arbeitsgruppe Verkehrsplanung, FGSV Verlag Köln (2002)

4. EMISIA S.A., http://www.emisia.com/copert

5. Infras: Handbuch für Emissionsfaktoren des Straßenverkehrs 1980-2010, Version 3.1. Bern (2010)

6. EMISIA S.A.: FLEETS Database online, http://www.emisia.com/tools/FLEETS.html 